\title{
Observational study of multiple myeloma in Latin America
}

\author{
Vania T. M. Hungria ${ }^{1}$ - Angelo Maiolino ${ }^{2}$ - Gracia Martinez $^{3}$. \\ Gislaine Oliveira Duarte ${ }^{4} \cdot$ Rosane Bittencourt $^{5} \cdot$ Lygia Peters $^{6} \cdot$ Gisele Colleoni $^{7}$. \\ Luciana C. O. Oliveira $^{8}$ - Edvan Crusoé ${ }^{9}$ Érika O. D. M. Coelho ${ }^{10}$. \\ Ricardo Pasquini ${ }^{11} \cdot$ Sílvia M. M. Magalhães ${ }^{12} \cdot$ Renata Nunes $^{13} \cdot$ Jorge V. Pinto Neto $^{14}$. \\ Rosa Malena O. Faria ${ }^{15}$. Mair Souza ${ }^{16}$. Nelson Hamerschlak ${ }^{17}$. Dorotea Flantl ${ }^{18}$. \\ J. R. Navarro ${ }^{19}$ - Guillermo Conte ${ }^{20}$ - David Gomez-Almaguer ${ }^{21}$. \\ Guillermo Ruiz-Argüelles ${ }^{22}$ - Brian G. M. Durie ${ }^{23}$ - On behalf of the International \\ Myeloma Working Group Latin America
}

Received: 27 April 2016/Accepted: 19 September 2016 / Published online: 5 November 2016

(C) Springer-Verlag Berlin Heidelberg 2016

\begin{abstract}
Relatively little is known about the outcomes of multiple myeloma in Latin America, a world region where incorporation of novel agents is generally slow. In the current retrospective-prospective study, we aimed to describe the patterns of care and treatment results in five Latin American countries. Between April 2007 and October 2009, patients who had been diagnosed from January 2005 to December 2007 were registered at 23 institutions from Argentina, Brazil, Chile, Mexico, and Peru. We divided patients into two cohorts, according to transplantation eligibility, and analyzed them with regard to first-line treatment and overall survival (OS). We analyzed a total of 852 patients, $46.9 \%$ of whom were female. The median follow-up was 62 months.
\end{abstract}

Vania T. M. Hungria

hungria@dialdata.com.br

On behalf of the International Myeloma Working Group Latin America

Santa Casa Medical School, São Paulo, Brazil

2 Universidade Federal do Rio de Janeiro, Rio de Janeiro, Brazil

3 Universidade de São Paulo, São Paulo, Brazil

4 Universidade Estadual de Campinas, Campinas, Brazil

5 Universidade Federal do Rio Grande do Sul, Porto Alegre, Brazil

6 Centro de Pesquisas Oncológicas de Santa Catarina, Florianópolis, Brazil

7 Universidade Federal de São Paulo, São Paulo, Brazil

8 Medical School of Ribeirão Preto, Ribeirão Preto, Brazil

9 Universidade Federal da Bahia, Salvador, Brazil

Among transplantation-ineligible patients $(N=461)$, the mean age was 67.4 years, approximately one third of patients received a thalidomide-based treatment in the first line, and the median OS was 43.0 months. Transplantation-eligible patients $(N=391)$ had a mean age of 54.7 years and a median OS of 73.6 months. Autologous transplantation was performed in $58.6 \%$ of the patients for whom this procedure was initially planned and in only $26.9 \%$ of the overall patients. Our longterm results reflect the contemporary literature for patients with multiple myeloma treated with autologous transplantation and thalidomide-based regimens in clinical trials and observational studies. However, further efforts are needed to approve and incorporate novel agents in Latin American

10

11

12

13

23 Inc. Cedar-Sinai Outpatient Cancer Center, Los Angeles, CA, USA

HEMOPE, Recife, Brazil

Universidade Federal do Paraná, Curitiba, Brazil

Universidade Federal do Ceará, Fortaleza, Brazil

Hospital Brigadeiro, São Paulo, Brazil

Hospital de Base de Brasília, Distrito Federal, Brazil

Universidade Federal de Minas Gerais, Belo Horizonte, Brazil

Fundação Dr Amaral de Carvalho, Jaú, Brazil

Hospital Albert Einstein, São Paulo, Brazil

Hospital Italiano, Buenos Aires, Argentina

Hospital Edgardo Rebagliati, Lima, Peru

Universidad de Chile, Santiago, Chile

Universidad Autonoma de Nuevo Leon, Monterrey, Mexico

Centro de Hematologia y Medicina Interna de Puebla, 
countries, as well as to increase access to transplantation, in order to achieve the expected improvements in patient outcomes.

Keywords Multiple myeloma $\cdot$ Survival analysis · Thalidomide $\cdot$ Transplantation $\cdot$ Autologous

\section{Introduction}

Multiple myeloma is thought to account for nearly $1 \%$ of all cancers and $15 \%$ of hematologic malignancies $[1,2]$. However, its incidence varies in different countries and ethnic groups. It is well known, for example, that the incidence of multiple myeloma is higher in blacks than in whites [3]. Conversely, the incidence of multiple myeloma is lower in Asia than in the USA and Western Europe; nevertheless, a progressive increase in the incidence of multiple myeloma has been reported in some Asian countries [4]. The clinical features of multiple myeloma may also differ across world regions, and it is important to characterize the profile of this disease in different geographic locations [5]. Finally, patient outcomes may also vary across countries and world regions as a result of differing biological features, stage at presentation, and availability of novel therapies.

Relatively little is known about the incidence, clinical features, and outcomes of multiple myeloma in Latin America, a world region where relatively free miscegenation of populations of Native American, Mediterranean, and African ancestry has occurred for several centuries. In Latin America, novel therapies tend to be approved at later stages than in the USA or European countries. As a result, access to agents such as bortezomib and lenalidomide is usually limited under the current public health systems of many Latin American countries. On the other hand, thalidomide has been available for patients with multiple myeloma and at a low cost in many of these countries for several years. In a previous study, we retrospectively assessed 1112 patients with multiple myeloma diagnosed in Brazil between 1998 and 2004, and our results suggested that the majority of the patients in this country present with advanced disease upon diagnosis [6]. Moreover, that study confirmed the prognostic utility of the International Staging System (ISS) [7] among these patients. Finally, the study demonstrated that the registration of patients with multiple myeloma is feasible in Brazilian institutions that are highly motivated. This led us to design the current observational study, with the main goals of gaining further insight about multiple myeloma by including other Latin American countries, describing the patterns of care and treatment results in this world region, and allowing participating investigators to share information on the demographic and clinical features of their patients.

\section{Methods}

\section{Study design and oversight}

This was an international, multicenter, retrospective-prospective study conducted under the auspices of the Latin American International Myeloma Foundation. The protocol for the study was approved by the institutional review board of all participating institutions, and written informed consent was obtained from all patients. Treatment and patient evaluation were left to the discretion of the participating investigators and local standards of care. The initial goal was to enroll approximately 1500 patients from nearly 30 centers from seven Latin American countries (Argentina, Brazil, Chile, Colombia, Mexico, Peru, and Venezuela). Patient registration was done between April 2007 and October 2009, and eligible patients were those diagnosed between 1 January 2005 and 31 December 2007. Therefore, for the majority of patients, registration was done in a retrospective fashion, whereas follow-up was prospective until the time of the database lock, on 31 December 2012. A follow-up period of at least 5 years for each patient was foreseen, unless death or loss to follow-up ensued before.

\section{Selection criteria and collected data}

Participating institutions were selected on the basis of clinical expertise and expected accrual. The chief inclusion criterion was the presence of multiple myeloma according to the International Myeloma Working Group criteria [8], and patients with any plasma cell- or immunoglobulin-related disorders other than multiple myeloma were excluded. None of the patients enrolled in the current study participated in our previous study [6]. Demographic and clinical data were gathered from institutional charts by locally designated individuals and were entered into a central, web-based registry specifically designed for the study. Such data included the date of diagnosis, the stage according to the Durie-Salmon staging (DSS) system [9] and the ISS [7], the type of monoclonal component, the results of relevant baseline laboratory tests, the eligibility for autologous transplantation, the types and dates of treatments administered, and the date of the last follow-up or death. No data on toxicity or quality of life were collected for the study. Data monitoring and analysis were performed by a contract research organization under the supervision of study investigators and sponsors, and the authors vouch for the full contents of the manuscript, which was drafted and reviewed without any involvement with commercial sponsors.

\section{Statistical analysis}

For the statistical analysis, two populations were defined on the basis of eligibility for transplantation. Transplantationineligible patients were analyzed as such (none of the patients 
who were initially ineligible underwent transplantation). On the other hand, not all the transplantation-eligible patients underwent transplantation for various reasons that were not investigated; however, these patients were analyzed in the transplantation-eligible group, along with those undergoing transplantation.

Overall survival (OS), the primary outcome measure, was defined as the time elapsed between the first day of the first treatment administered to each patient and death from any cause, with censoring of patients who were alive in the last follow-up. OS was estimated by the Kaplan-Meier method, and differences between groups were compared using the $\log$-rank test. For the analysis of OS according to receipt of transplantation, the landmark method was used [10]. The median follow-up was estimated using the reverse Kaplan-Meier method [11]. Two-sided $p$ values $<0.05$ were considered statistically significant. Statistical analysis was performed using MedCalc $\AA$, version 11.0.0.0 (Mariakerke, Belgium).

\section{Results}

\section{Overview of patient accrual and follow-up}

A total of 852 patients diagnosed between January 2005 and December 2007 were registered between April 2007 and October 2009 at 23 institutions from Argentina, Brazil, Chile, Mexico, and Peru. The median follow-up on survival for the 852 patients was 62 months.

\section{Transplantation-ineligible patients}

Of the 852 patients, 461 (54.1\%) were ineligible for autologous transplantation. Table 1 presents their main demographic and clinical features, as well as their country of enrollment. The mean age was 67.4 years, nearly two thirds of the patients had DSS stage III disease, and $37.9 \%$ had ISS stage III disease. Nearly one third of the patients received a thalidomidebased treatment in the first line, and subsequent lines of therapies were administered to nearly half of the patients in the second line and $15 \%$ in the third line. None of the patients received either bortezomib or lenalidomide in the first line. The available data allowed for the analysis of OS in 441 of 461 transplantation-ineligible patients. Their survival experience is depicted in Fig. 1. Their median OS was 43.0 months, with an estimated survival rate of approximately $38 \%$ at 5 years. With regard to the use of thalidomide in the firstline regimen, there was no significant difference in the OS according to this use (medians of 42.8 and 44.1 months for patients with and without thalidomide, respectively; hazard ratio, $0.91 ; 95 \%$ confidence interval, $0.68-1.21 ; p=0.508$ ).

\section{Transplantation-eligible patients}

Of the 852 patients, 391 (45.9\%) were initially eligible for autologous transplantation upon diagnosis. Table 1 presents their main features. Their mean age was 54.7 years, nearly 72 $\%$ of the patients had DSS stage III disease, and $30 \%$ had ISS stage III disease. Transplantation-ineligible patients were on average nearly 13 years older, more likely to be female, and more likely to present with ISS stage III disease than transplantation-eligible patients. The most common first-line regimens were vincristine/doxorubicin/dexamethasone or an equivalent regimen, which were administered to nearly two thirds of patients; thalidomide/dexamethasone was used in approximately $20 \%$ of the patients, with all other regimens comprising only $16.9 \%$ of the cases (none of the patients received either bortezomib or lenalidomide in the first line). Autologous transplantation was actually administered to only $58.6 \%$ of patients for whom this procedure was planned and in $26.9 \%$ of the 852 overall patients. Subsequent lines of therapies were administered to $64.2 \%$ of the transplantation-eligible patients in the second line and $25.3 \%$ in the third line. Of note is that bortezomib and/or lenalidomide were given to less than $10 \%$ of these patients as rescue therapy. Once again, there was no significant difference in the OS according to the use of thalidomide in the first-line regimen (medians of 60.5 and 79.5 months for patients with and without the agent, respectively; hazard ratio, 1.12; $95 \%$ confidence interval, $0.75-1.68$; $p=0.570)$.

The available data allowed for the analysis of OS in 388 of 391 transplantation-eligible patients. Their survival experience is depicted in Fig. 2. Patient median OS was 73.6 months, with an estimated survival rate of approximately $56 \%$ at 5 years. Considering patients who underwent transplantation, the procedure was done after a median of 9.4 months (interquartile range, 6.8-13.8 months) after treatment initiation. Among transplantation-eligible patients who were alive at 12 months $(N=320)$, a landmark analysis was performed comparing the OS of those who indeed underwent the procedure before the landmark $(N=133)$ and those who, despite an initial recommendation, did not undergo transplantation or had it after the landmark of 12 months $(N=187)$. The OS from the landmark did not differ significantly between patients who did and who did not undergo transplantation (median OS not reached in both groups; hazard ratio, $0.85 ; 95 \%$ confidence interval, $0.59-1.23 ; p=0.398$ ).

\section{Baseline prognostic factors overall}

For the overall patient sample, we assessed conventional prognostic factors for OS. There were statistically significant differences in OS according to DSS stage (median OS not reached, 73.3 and 47.8 months for stages I, II, and III, respectively; $p<0.001$ ), ISS stage (median OS of 79.7, 56.5, and 
Table 1 Selected baseline characteristics of patients overall and according to eligibility for transplantation

\begin{tabular}{|c|c|c|c|}
\hline Characteristics & $\begin{array}{l}\text { Transplantation-ineligible } \\
\text { patients } \\
\text { Value or } N(\%)\end{array}$ & $\begin{array}{l}\text { Transplantation-eligible } \\
\text { patients } \\
\text { Value or } N(\%)\end{array}$ & $\begin{array}{l}\text { All patients } \\
\text { Value or } N(\%)\end{array}$ \\
\hline Age & $N=460$ & $N=390$ & $N=850$ \\
\hline Median (range, years) & $68.6(31.3-97.9)$ & $55(25.9-73.1)$ & $60.9(25.9-97.9)$ \\
\hline $\begin{array}{l}\text { Mean (standard deviation, } \\
\text { years) }\end{array}$ & $67.4(11.2)$ & $54.7(8.2)$ & $61.6(11.8)$ \\
\hline Sex & $N=461$ & $N=391$ & $N=852$ \\
\hline Female & $233(50.5)$ & $167(42.7)$ & $400(46.9)$ \\
\hline Male & $228(49.5)$ & $224(57.3)$ & $452(53.1)$ \\
\hline Ethnicity & $N=461$ & $N=391$ & $N=852$ \\
\hline $\begin{array}{l}\text { Mixed ancestry, including } \\
\text { mulatto }\end{array}$ & $171(37.1)$ & $185(47.3)$ & $356(41.8)$ \\
\hline European ancestry & $140(30.4)$ & $103(26.3)$ & $243(28.5)$ \\
\hline Native American ancestry & $44(9.5)$ & $59(15.1)$ & $103(12.1)$ \\
\hline African ancestry & $32(6.9)$ & $19(4.9)$ & $51(6.0)$ \\
\hline Other or unknown & $74(16.0)$ & $25(6.4)$ & 99 (11.6) \\
\hline Country of enrolment & $N=461$ & $N=391$ & $N=852$ \\
\hline Argentina & $44(9.5)$ & $13(3.3)$ & $57(6.7)$ \\
\hline Brazil & $330(71.6)$ & $347(88.7)$ & $677(79.5)$ \\
\hline Chile & $32(6.9)$ & $11(2.8)$ & $43(5.0)$ \\
\hline Mexico & $22(4.8)$ & 0 & $22(2.6)$ \\
\hline Peru & $33(7.2)$ & $20(5.1)$ & $53(6.2)$ \\
\hline Monoclonal component & $N=420$ & $N=376$ & $N=796$ \\
\hline $\operatorname{IgG}$ & $246(58.6)$ & $203(54.0)$ & $449(56.4)$ \\
\hline $\operatorname{Ig} \mathrm{A}$ & $102(24.3)$ & $85(22.6)$ & $187(23.5)$ \\
\hline $\operatorname{IgM}$ & $5(1.2)$ & $2(0.5)$ & $7(0.9)$ \\
\hline Light chain only & $56(13.3)$ & $75(19.9)$ & $131(16.5)$ \\
\hline Non-secretory & $11(2.6)$ & $11(2.9)$ & $22(2.8)$ \\
\hline Bone lesions & $N=436$ & $N=372$ & $N=808$ \\
\hline Absent & $61(14.0)$ & $33(8.9)$ & 94 (11.6) \\
\hline Present & $375(86.0)$ & $339(91.1)$ & $714(88.4)$ \\
\hline Durie-Salmon stage & $N=434$ & $N=377$ & $N=811$ \\
\hline I & $61(14.1)$ & $16(4.2)$ & $77(9.5)$ \\
\hline II & $84(19.3)$ & $91(24.1)$ & 175 (21.6) \\
\hline III & $289(66.6)$ & $270(71.6)$ & $559(68.9)$ \\
\hline $\begin{array}{l}\text { International Staging System } \\
\text { stage }\end{array}$ & $N=380$ & $N=333$ & $N=713$ \\
\hline I & $101(26.6)$ & $108(32.4)$ & $209(29.3)$ \\
\hline II & $135(35.5)$ & $125(37.5)$ & $260(36.5)$ \\
\hline III & $144(37.9)$ & $100(30.0)$ & $244(34.2)$ \\
\hline Creatinine & $N=459$ & $N=374$ & $N=833$ \\
\hline$\leq 2 \mathrm{mg} / \mathrm{dL}$ & $336(73.2)$ & 291 (77.8) & $627(75.3)$ \\
\hline$>2 \mathrm{mg} / \mathrm{dL}$ & $123(26.8)$ & $83(22.2)$ & $206(24.7)$ \\
\hline Hypercalcemia & $N=428$ & $N=356$ & $N=784$ \\
\hline Absent & 327 (76.4) & $268(75.3)$ & 595 (75.9) \\
\hline Present & $101(23.6)$ & 88 (24.7) & $189(24.1)$ \\
\hline
\end{tabular}

Ig immunoglobulin
33.9 months for stages I, II, and III, respectively; $p<0.001$ ), the presence of hypercalcemia (median OS of 61.6 and 31.3 months for patients with and without hypercalcemia, respectively; $p<0.001$ ), and the presence of renal impairment (median OS of 59.0 and 34.1 months for patients with serum creatinine $\leq 2$ and $>2 \mathrm{mg} / \mathrm{dL}$, respectively; $p<0.001$ ). 
Fig. 1 Overall survival of transplantation-ineligible patients (tick marks represent censoring)

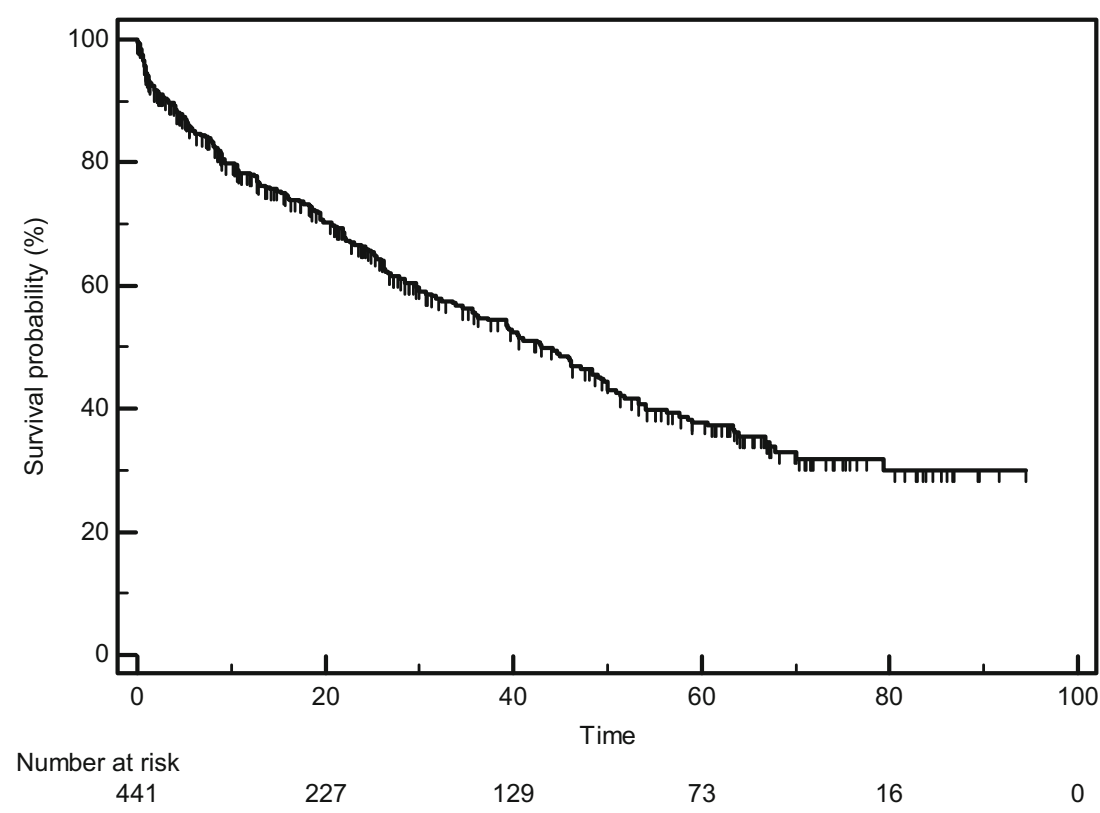

\section{Discussion}

Paradigm shifts in the treatment of multiple myeloma, such as high-dose chemotherapy rescued by autologous transplantation and the use of immunomodulators and proteasome inhibitors, have allowed for unprecedented improvements in the short- and long-term treatment results for these patients [12]. Unfortunately, transplantation is not feasible in approximately three quarters of the patients, either because of advanced age or comorbidities. On the other hand, the efficacy of immunomodulators and proteasome inhibitors, when used as front-line therapy, has called into question the role of transplantation even when it is feasible [13]. However, recent interim results from a randomized trial suggest that transplantation should remain a standard of care among eligible patients [14]. Moreover, there is no evidence of decreased utilization of transplantation in the USA and Europe up to 2009/2010 [15, 16]. In addition to transplantation and novel agents, maintenance strategies are also reshaping the approach to multiple myeloma both in the transplantation setting and for transplant-ineligible patients [17-20]. As a result of these improvements, the expected median OS among patients with newly diagnosed multiple myeloma is often close to 5 years among transplant-
Fig. 2 Overall survival of transplantation-eligible patients (tick marks represent censoring)

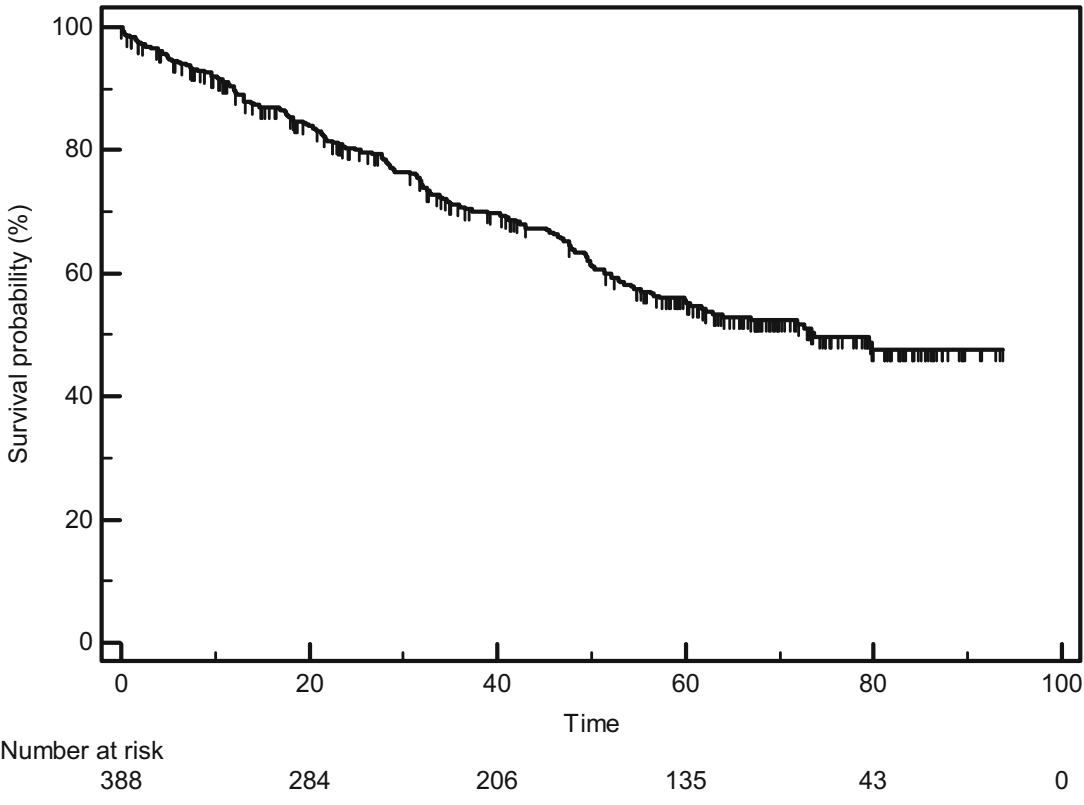


ineligible patients treated with current regimens [19-22] and typically longer in the transplantation setting [17, 18], in which the adoption of an "optimal strategy" is expected to produce survival rates approaching $80 \%$ at 5 years [12].

To the best of our knowledge, this is the first study including patients with multiple myeloma from several Latin American countries followed prospectively. Unfortunately, not all countries that initially committed to participation were able to enroll patients, and the overall accrual was lower than the expected in some countries. Despite these limitations, we believe our results are representative of the experience of public institutions in Latin America. On the other hand, the Latin American experience at private institutions, where a minority of the population is treated, may well differ from the results reported herein because there is a gap between public and private institutions with regard to the resources that are available for the management of various diseases in Latin America. To our knowledge, the potential prognostic impact of such disparities has not been investigated in this world region for multiple myeloma, but studies in breast and prostate cancer have identified differences in stage at presentation as a possible explanation for worsened outcomes in public institutions in Brazil [23, 24]. Given these features of our world region, we believe it is important to assess treatment results on an ongoing basis, while work continues to be done towards improving access to novel therapies.

The demographic and baseline clinical features of our patients can be contrasted with those reported by Kim et al., who have analyzed 3405 patients from various Asian countries and found a median age of 62 years, $55.6 \%$ of males, serum creatinine $<2 \mathrm{mg} / \mathrm{dL}$ in $76.6 \%$ of patients, and $44 \%$ of ISS stage III disease [5]. As shown in Table 1, Latin American patients had mostly similar demographic features, but a lower percentage of ISS stage III disease (34.2\%) and somewhat different distributions of other well-known prognostic factors, such as hypercalcemia. Whether these different distributions represent different underlying biology or factors related to the pattern of referral is not clear. It should be noted that the Asian series comprises patients diagnosed between 1986 and 2011, and changes in diagnostic modalities may thus underlie some of the observed differences.

The median age is low, in comparison with published studies, both for our transplant-ineligible (68.5 years) and transplant-eligible (55 years) patients. This finding was already evident in our previous, non-overlapping series [6]. Corresponding medians in published trials ranged between 71 and 72.5 years $[22,25]$ and between 57 and 58 years [14, 18], respectively. In our study, transplant eligibility was ascertained locally; therefore, there were no centrally determined criteria that could be verified or adjudicated. However, in the investigators' meeting conducted before data collection, the clinical condition of the patients was agreed upon as a major determinant of transplant eligibility. On the other hand, we have reasons to believe that delayed referral plays a central role in determining patient fitness in our region, but the extent to which this was indeed the case cannot be ascertained with the available data.

Our OS results for transplantation-ineligible patients (median OS of 43.0 months, with an estimated 5-year OS of nearly 38 $\%$ ) are within the range reported in the literature for randomized trials among elderly or transplantation-ineligible patients treated with thalidomide-based regimens in the first line [21, 22, 26, 27]. On the other hand, the subsequent addition of bortezomib to the first-line regimen increased the median OS of these patients to 56 months [25]. Notably, many countries have not been able to incorporate some of the novel agents as front-line therapy against multiple myeloma due to cost or local unavailability. Indeed, less than $10 \%$ of patients participating in the current study ever received bortezomib or lenalidomide as a component of therapy after the first relapse. This is the case of Latin American countries, most of which only have thalidomide available at public institutions. Although the similarity between our results and those reported in the literature on thalidomidebased first-line regimens is reassuring, it is conceivable that the outcomes of our patients could be further improved by the availability of novel therapies, to be used in the first line or after disease progression, and which have probably contributed to the OS experience of patients from some of the more recently published trials [28].

Our OS results for transplantation-eligible patients (median OS of 73.6 months) also compare favorably to results reported in the literature, even considering more recent trials [17-19]. Of note is that the median OS was not reached for the subset of patients actually undergoing transplantation, whereas this median was 52.1 months among those who did not receive transplantation. Therefore, it is possible that transplantation of a higher proportion of patients would lead to improved results. In the current study, $45.9 \%$ of patients were initially eligible to autologous transplantation, and only $26.9 \%$ of all patients in fact underwent such procedure. In the Mayo Clinic experience from 2001 to $2010,37 \%$ of patients with multiple myeloma eventually received an autologous transplantation [28]. It is also noteworthy that only $58.6 \%$ of initially transplantation-eligible patients in fact underwent the procedure. Unfortunately, no data were captured that would allow an assessment of the reasons why individual patients were eventually not treated with transplantation. Nevertheless, the most frequent explanation is likely to be loss to follow-up. In addition, an unduly long time between diagnosis and referral for autologous transplantation has been highlighted as a common problem in Brazil [29]. Thus, earlier diagnosis and referral, as well as increased use of autologous transplantation, could improve patient outcomes, something that remains to be investigated. Moreover, since most novel agents are not yet available at public institutions from many Latin American countries, it is possible that our results could be further improved by the addition of such agents to the therapeutic arsenal. 
Our OS results can also be compared with those reported from Asia in a similar, albeit larger, observational study [5]. In that study, $19.8 \%$ of patients with multiple myeloma underwent transplantation, and these patients had a median OS of 79 months. Conversely, the median OS was 41 months for the $80.2 \%$ of patients that did not receive transplantation. In our study, the median survival of transplantation-eligible patients (73.6 months) was longer than that of transplantationineligible individuals (43 months), but these two groups represent populations with different prognostic factors. In a landmark survival analysis of transplantation-eligible patients, actual receipt of transplantation before the 12-month landmark had no significant prognostic role in univariable analysis. It should be noted that this analysis is exploratory and observational in nature and does not question the role of autologous transplantation in multiple myeloma.

In recent years, cost has emerged as an overarching concern in the treatment of solid tumors and hematologic malignancies. Cost considerations are especially acute for incurable tumors that mostly affect elderly populations, such as multiple myeloma. Unfortunately, no studies have been published assessing the comparative costs or cost-effectiveness of competing treatment strategies for Latin American patients with multiple myeloma. Given the limited healthcare resources typically found in this world region, especially at publicly funded institutions, further studies should attempt to investigate the comparative merit of the incorporation of novel agents and of increasing the use of transplantation. Both of these strategies will probably be necessary towards moving in the direction of treatment goals that are currently expected in developed countries [12]. A definitive answer to many important questions that remain in the treatment of multiple myeloma can only come from randomized trials; unfortunately, such trials are unlikely to be conducted in a world region where there is comparatively little support for investigator-initiated trials.

\section{Compliance with ethical standards}

Conflict of interest The authors declare that they have no conflict of interest.

\section{References}

1. Siegel R, Ma J, Zou Z, Jemal A (2014) Cancer statistics, 2014. CA Cancer J Clin 64:9-29

2. Blade J, Cibeira MT, Fernandez De Larrea C, Rosinol L (2010) Multiple myeloma. Ann Oncol 21(Suppl 7):vii313-vii319

3. Benjamin M, Reddy S, Brawley OW (2003) Myeloma and race: a review of the literature. Cancer Metastasis Rev 22:87-93

4. Huang SY, Yao M, Tang JL et al (2007) Epidemiology of multiple myeloma in Taiwan: increasing incidence for the past 25 years and higher prevalence of extramedullary myeloma in patients younger than 55 years. Cancer 110:896-905

5. Kim K, Lee JH, Kim JS et al (2014) Clinical profiles of multiple myeloma in Asia - an Asian Myeloma Network study. Am J Hematol 89:751-756

6. Hungria VT, Maiolino A, Martinez G et al (2008) Confirmation of the utility of the International Staging System and identification of a unique pattern of disease in Brazilian patients with multiple myeloma. Haematologica 93:791-792

7. Greipp PR, San Miguel J, Durie BG et al (2005) International staging system for multiple myeloma. J Clin Oncol 23:3412-3420

8. International Myeloma Working Group (2003) Criteria for the classification of monoclonal gammopathies, multiple myeloma and related disorders: a report of the international myeloma working group. Br J Haematol 121:749-757

9. Durie BG, Salmon SE (1975) A clinical staging system for multiple myeloma. Correlation of measured myeloma cell mass with presenting clinical features, response to treatment, and survival. Cancer 36:842-854

10. Anderson JR, Cain KC, Gelber RD (1983) Analysis of survival by tumor response. J Clin Oncol 1:710-719

11. Schemper M, Smith TL (1996) A note on quantifying follow-up in studies of failure time. Control Clin Trials 17:343-346

12. Moreau P, Attal M, Facon T (2015) Frontline therapy of multiple myeloma. Blood 125:3076-3084

13. Preeshagul IR, Van Besien K, Mark TM (2014) Controversies in multiple myeloma: to transplant or not? Curr Hematol Malig Rep 9: 360-367

14. Attal M, Lauwers-Cances V, Hulin C et al. (2015) Autologous transplantation for multiple myeloma in the era of new drugs: a phase III study of the Intergroupe Francophone du Myelome (IFM/DFCI 2009 Trial). Blood 126(23):391

15. Costa LJ, Zhang MJ, Zhong X et al (2013) Trends in utilization and outcomes of autologous transplantation as early therapy for multiple myeloma. Biol Blood Marrow Transplant 19: $1615-1624$

16. Auner HW, Szydlo R, Hoek J et al (2015) Trends in autologous hematopoietic cell transplantation for multiple myeloma in Europe: increased use and improved outcomes in elderly patients in recent years. Bone Marrow Transplant 50:209-215

17. Attal M, Lauwers-Cances V, Marit G et al (2012) Lenalidomide maintenance after stem-cell transplantation for multiple myeloma. N Engl J Med 366:1782-1791

18. Palumbo A, Cavallo F, Gay F et al (2014) Autologous transplantation and maintenance therapy in multiple myeloma. N Engl J Med 371:895-905

19. Palumbo A, Hajek R, Delforge M et al (2012) Continuous lenalidomide treatment for newly diagnosed multiple myeloma. $\mathrm{N}$ Engl J Med 366:1759-1769

20. Benboubker L, Dimopoulos MA, Dispenzieri A et al (2014) Lenalidomide and dexamethasone in transplant-ineligible patients with myeloma. N Engl J Med 371:906-917

21. Facon T, Mary JY, Hulin C et al (2007) Melphalan and prednisone plus thalidomide versus melphalan and prednisone alone or reduced-intensity autologous stem cell transplantation in elderly patients with multiple myeloma (IFM 99-06): a randomised trial. Lancet 370:1209-1218

22. Palumbo A, Bringhen S, Caravita Tet al (2006) Oral melphalan and prednisone chemotherapy plus thalidomide compared with melphalan and prednisone alone in elderly patients with multiple myeloma: randomised controlled trial. Lancet 367:825-831

23. Liedke PE, Finkelstein DM, Szymonifka J et al (2014) Outcomes of breast cancer in Brazil related to health care coverage: a retrospective cohort study. Cancer Epidemiol Biomarkers Prev 23:126-133

24. Nardi AC, Reis RB, Zequi Sde C, Nardozza A Jr (2012) Comparison of the epidemiologic features and patterns of initial care for prostate 
cancer between public and private institutions: a survey by the Brazilian Society of Urology. Int Braz J Urol 38:155-164; discussion 164-156

25. San Miguel JF, Schlag R, Khuageva NK et al (2008) Bortezomib plus melphalan and prednisone for initial treatment of multiple myeloma. N Engl J Med 359:906-917

26. Fayers PM, Palumbo A, Hulin C et al (2011) Thalidomide for previously untreated elderly patients with multiple myeloma: metaanalysis of 1685 individual patient data from 6 randomized clinical trials. Blood 118:1239-1247
27. Hungria VT, Crusoe EQ, Maiolino A et al. (2016) Phase 3 trial of three thalidomide-containing regimens in patients with newly diagnosed multiple myeloma not transplant-eligible. Ann Hematol 95: 271-278

28. Kumar SK, Dispenzieri A, Lacy MQ et al (2014) Continued improvement in survival in multiple myeloma: changes in early mortality and outcomes in older patients. Leukemia 28:1122-1128

29. Saccilotto IC, Bittencourt RI, Fischer CC et al (2015) Efficacy of a referral center for patient-centered care in multiple myeloma: a cohort study. BMC Health Serv Res 15:455 